\title{
LOCALLY DELIVERED ETHYL-2,5-DIHYDROXYBENZOATE USING 3D PRINTED BONE IMPLANT FOR PROMOTION OF BONE REGENERATION IN A OSTEOPOROTIC ANIMAL MODEL
}

\author{
B-J. Kwon ${ }^{1}$, G.M. Seon ${ }^{1,2}$, M.H. Lee ${ }^{1}$, M-A. Koo ${ }^{1,2}$, M.S. Kim ${ }^{1,2}$, D. Kim ${ }^{1}$, J-J. Han ${ }^{3}$, D. Kim \\ J. Kim ${ }^{5}$ and J-C. Park ${ }^{1,2, *}$
}

\author{
${ }^{1}$ Cellbiocontrol Laboratory, Department of Medical Engineering, Yonsei University College of Medicine, \\ Seoul, 03722, Republic of Korea \\ ${ }^{2}$ Brain Korea 21 PLUS Project for Medical Science, Yonsei University College of Medicine, \\ Seoul, 03722, Republic of Korea \\ ${ }^{3}$ Cellsafe Ltd., Gyeonggi-do, 16499, Republic of Korea \\ ${ }^{4}$ InssTec Inc., Daejeon, 34109, Republic of Korea \\ ${ }^{5}$ Department of Biomedical Materials, Konyang University, Daejeon, 35365, Republic of Korea
}

\begin{abstract}
Osteoporosis is a disease characterized by low bone mass, most commonly caused by an increase in bone resorption that is not matched by sufficient bone formation. The most common complications of postmenopausal osteoporosis are bone-related defects and fractures. Fracture healing is a multifactorial bone regeneration process, influenced by both biological and mechanical factors related to age, osteoporosis and stability of the osteosynthesis. During the treatment of bone defects in osteoporotic conditions, imbalanced bone remodeling is the leading cause for implant failure. To overcome these problems, ethyl-2,5dihydroxybenzoate (E-2,5-DHB), a drug that promotes bone formation and inhibits bone resorption, was used. E-2,5-DHB-incorporating titanium (Ti) implants using poly(lactic-co-glycolic acid) (PLGA) coating for local delivery of E-2,5-DHB were developed and the effects on bone healing of femoral defects were evaluated in an osteoporotic model. The release of E-2,5-DHB resulted in decreased bone resorption and increased bone formation around the implant. Thus, it was confirmed that, in the osteoporotic model, bone healing was increased and implant fixation was enhanced. These results suggested that E-2,5-DHB-coated Ti implants have great potential as an ultimate local drug delivery system for bone tissue scaffolds.
\end{abstract}

Keywords: Osteoporosis, bone formation, bone resorption, bone fracture healing, ethyl-2,5-dihydroxybenzoate.

*Address for correspondence: Jong-Chul Park, PhD, Cellbiocontrol Laboratory, Department of Medical Engineering, Yonsei University College of Medicine, Seoul, 03722, Republic of Korea.

Telephone: +82 222281917 Fax: +8223639923 Email: parkjc@yuhs.ac

\section{Introduction}

Osteoporosis is a global emerging healthcare issue and a socio-economic problem characterized by reduced bone mass, weak bone strength and microarchitectural aggravation in the cortical and trabecular skeleton. These features lead to skeletal fragility and sensitivity to fractures. Osteoporosis is an age-related disease caused by a disproportion between osteogenic bone formation and osteoclastic bone resorption, which is often the result of postmenopausal estrogen deficiency (Pei and Tontonoz, 2004; Rodan and Martin, 2000).

In recent years, fracture healing has been widely studied, but less attention has been placed on osteoporotic fractures. Since osteoporosis-related fractures generally occur at long bone sites subjected to loading stress, mechanical intervention may affect the healing process. A few studies report the therapeutic effect of local implantation of biomimetic scaffolds during osteoporotic defect regeneration (Cheng et al., 2013; Zhang et al., 2012). Fractures of osteoporotic bones present diverse challenges, including a large proportion of fixation failure and impaired healing capability, which make their medical management challenging (Kyllönen et al., 2015). Osteoporotic bone fracture, not implant breakage, is the primary cause of failure of internal fixation. Because bone mineral density associates with the ability to hold screws, osteoporotic bone often lacks the strength to hold plates and screws securely (Alho, 1993; Sjostedt et al., 1994; Strømsøe et al., 1993). The major challenges in the treatment of osteoporotic fractures are stable internal fixation, without implant loosening, and subsequent successful bone healing. However, alterations in bone turnover, bone tissue quality and delays in fracture healing are some of the well-known key issues that must be addressed 
by clinicians and researchers to improve treatment outcomes for patients with osteoporotic fractures (Barrios et al., 1993; Gardner, 2004).

The discovery of osteopromotive and inductive agents, with the potential to stimulate bone formation, has raised hope for the augmentation of osteoporotic fracture healing. Local release of a bone anabolic agent at the site of an osteoporotic fracture would potentially facilitate a rapid increase in bone strength and quality, as well as reduce the bone healing period and prevent the development of problematic non-union. Local delivery is expected to increase the utility and efficacy of bone anabolic or antiresorptive drugs and decrease adverse effects related to their systemic administration.

Ethyl-2,5-dihydroxybenzoate (E-2,5-DHB) is a derivative of ethyl-3,4-dihydroxybenzoate (E-3,4-DHB), which is an element of the Rubus coreanus extract. Rubus coreanus total extract is a natural bioactive substance with bone-protective effects during postmenopausal osteoporosis in ovariectomized rats (Do et al., 2008). A natural bioactive substance, it has been considered as a biomedical material due to its good biocompatibility and various bio-active characteristics. E-3,4-DHB also improves osteoinductive activity by stimulating osteoblast differentiation and reduces bone resorption by inhibiting osteoclast differentiation (Kwon et al., 2014). However, the effect of E-2,5-DHB on bone healing in osteoporotic conditions has yet to be clearly defined. Therefore, it was hypothesized that E-2,5-DHB, with its dual effects on osteoblasts and osteoclasts, would promote bone defect healing in an osteoporotic model.

Titanium (Ti) and its alloys are extensively used as implant materials in the orthopedic and dental fields because of their excellent biocompatibility and mechanical properties (Long et al., 1998). Ti facilitates new bone formation and provides long-lasting boneimplant stability (Ravichandran et al., 2012). Ti and its alloys are widely used as hard tissue replacements in artificial bones, joints and dental implants. As these types of replacements, their low elastic modulus is generally viewed as a biomechanical advantage because they can produce smaller stress shielding (Sáenz de Viteri and Fuentes, 2013). If several interconnected pores are introduced into $\mathrm{Ti}$ and its alloys, their elastic modulus decreases to a level similar to that of cancellous bone (Pattanayak et al., 2011). Porous bone scaffolds can be made by a variety of methods and selective laser melting (SLM) processes are used to produce porous bone substitutes (Mullen et al., 2009; Stamp et al., 2009; Thijs et al., 2010).

Our previous study reports whether E-2,5-DHB can be an effective material and whether it plays a role in inducing osteoblast differentiation and inhibiting osteoclast differentiation (Kwon et al., 2016). In the treatment of bone defects in osteoporotic conditions, imbalanced bone remodeling is the leading cause of implant failure. To overcome these problems, E-2,5-DHB, a drug that promotes bone formation and inhibits bone resorption, was used. In this study, an E-2,5-DHB-incorporating Ti implant using poly(lactic-co-glycolic acid) (PLGA) coating for local delivery of E-2,5-DHB was developed and its effects on bone healing of femoral defects in an osteoporotic rat model were evaluated.

\section{Materials and Methods}

\section{Materials}

The PLGA polymer (polylactic acid/polyglycolic acid $=50 / 50$ ) was obtained from Evonik (Birmingham, AL, USA). Acetone was obtained from Duksan Pure Chemicals Co., Ltd (Kyungki Do, Korea). E-2,5DHB was supplied by Cell Safe Ltd. (Kyungki Do, Korea). MC3T3-E1 mouse preosteoblast cells were purchased from Riken BRC cell bank (Tokyo, Japan). $\alpha$-modification minimum essential medium ( $\alpha$-MEM) and fetal bovine serum (FBS) were obtained from WelGENE (Daegu, Korea). Bradford assay kit was purchased from Bio-Rad (Hercules, CA, USA).

\section{Three-dimensional printed porous titanium implants containing E-2,5-DHB}

Three-dimensional (3D) printed porous Ti implants were produced by a powder bed fusion (PBF) metal 3D printer (SLM system: Metasys250; Winforsys, Kyungki Do, Korea) using Ti powder (Ti-6Al-4V; LPW-Ti64Gd5; LPW Technology Ltd., Sci-Tech,
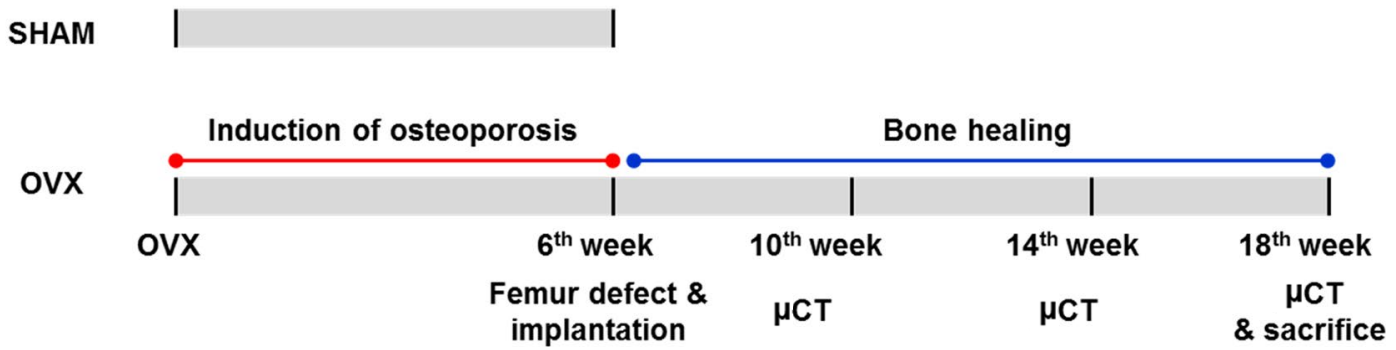

Fig. 1. Schematic diagram indicating the surgery time points for rats in the sham surgery (SHAM) and ovariectomy (OVX) groups. 6 weeks after ovariectomy, establishment of the osteoporotic model was confirmed in the OVX rats, using SHAM rats as control. Also at 6 weeks, femur defect drilling was performed in the OVX rats, followed by implantation with 3D printed Ti implants. After 4, 8 and 12 weeks, OVX rats were sacrificed and femur specimens were removed for assessment. 
Table 1. Ethyl-2,5-dihydroxybenzoate (E-2,5-DHB) concentration in the E-2,5-DHB-incorporating PLGAcoated titanium implants. Data are shown as mean \pm SD.

\begin{tabular}{|l|c|c|}
\hline & \multicolumn{2}{|c|}{ Initial E-2,5-DHB concentration (mg) } \\
\hline & 1 & 2 \\
\hline Measured concentration $(\mathrm{mg})$ & $1.1 \pm 0.7$ & $1.9 \pm 2.2$ \\
\hline
\end{tabular}

Daresbury, UK). The Ti implants were manufactured using an SLM system (Metasys250; Winforsys). This system contains a $\mathrm{Yb}$ fiber laser beam with a nominal diameter of 70 to $150 \mu \mathrm{m}$ and maximum power of $150 \mathrm{~W}$. Cylindrical specimens of $3 \mathrm{~mm}$ in diameter and height were manufactured. The porous Ti implants were solvent-cleansed by serial ultrasonic treatment in acetone, ethyl alcohol and de-ionized water. The 3D printed porous Ti implants were sterilized before coating by incubation in $70 \%(\mathrm{v} / \mathrm{v})$ ethanol for $30 \mathrm{~min}$ and then washed 5 times with distilled water. PLGA was dissolved in acetone to prepare $15 \%$ PLGA-acetone solutions. To accelerate its dissolution in acetone, PLGA was stirred for $1 \mathrm{~h}$. E-2,5-DHB in acetone was incorporated into the PLGA solution before coating. 3D printed porous Ti implants were immersed in the PLGA-acetone-E2,5-DHB solution for $5 \mathrm{~min}$ to force the solution into the porous structure. Then, the PLGA-coated 3D Ti implants were dried for $5 \mathrm{~d}$ to remove the residual acetone.

\section{Characterization of the E-2,5-DHB-incorporating PLGA-coated titanium implants}

Surface morphology of the Ti implants was observed with a field emission scanning electron microscope (FE-SEM S-800; Hitachi, Tokyo, Japan) using an accelerating voltage of $20 \mathrm{kV}$, as well as by microcomputed tomography ( $\mu \mathrm{CT}$ ) (NFR Polaris-G90; NanoFocus Ray Co., Jeonju, Korea).

E-2,5-DHB loading capacity was determined by measuring the concentration of coated E-2,5-DHB. The E-2,5-DHB-incorporating PLGA-coated Ti implants were immersed in DMSO. After vortexing, the solution was measured spectrophotometrically at $338 \mathrm{~nm}$. To determine the release kinetics of E-2,5-DHB from the Ti implants, the E-2,5-DHBincorporating PLGA-coated Ti implants were immersed in $1 \mathrm{~mL}$ of phosphate-buffered saline (PBS) solution at $37^{\circ} \mathrm{C}$. At predetermined time intervals of 1,3 and $5 \mathrm{~h}$ and $1,3,7,14,21$ and $28 \mathrm{~d}$, the supernatant was collected and replaced with fresh PBS solution. The amount of E-2,5-DHB released in the collected solution was measured spectrophotometrically at $240 \mathrm{~nm}$.

In vivo bone healing in an osteoporotic animal model using 3D printed titanium implants coated with PLGA incorporated with E-2,5-DHB

All animal experiments were performed in accordance with the Korean Food and Drug Administration guidelines. Protocols were reviewed and approved by the Institutional Animal Care and Use Committee of the Yonsei Laboratory Animal Research Center (Permit No. 2015-0120). All animals were kept at 20$26^{\circ} \mathrm{C}$ under a $12 \mathrm{~h} \mathrm{light/dark} \mathrm{cycle} \mathrm{and} \mathrm{allowed} \mathrm{food}$ and water ad libitum.

20 female Sprague-Dawley rats (3 months old), which underwent several procedures during the course of the experiment (Fig. 1), were acclimatized for 1 week before the experiments. The animals were anesthetized by an intramuscular injection of Zoletil (30 mg/kg; MNS Korea, Kyungki Do, Korea) and Rompun (10 mg/kg; MNS Korea). The abdominal cavity was accessed by a $20 \mathrm{~mm}$ midline dorsal skin incision, which allowed for blunt dissection of the connective tissue between the skin and the muscular layer of the abdominal wall. The ovary was located in a fat pad and gently pulled outside the abdominal cavity. Two ligatures were placed with absorbable 4-0 sutures. The ovaries were cut and removed and the uterine horns were placed back into the abdominal cavity. The abdominal muscles were closed with absorbable 4-0 sutures using a continuous suture technique, after which the skin was sutured with multiple single absorbable sutures. The animals were administered $1 \mathrm{mg} / \mathrm{kg}$ meloxicam and $5 \mathrm{mg} /$ $\mathrm{kg}$ gentamicin subcutaneously for postoperative analgesia and infection prophylaxis, respectively. The SHAM-operated group underwent the same surgical procedure as the ovariectomy (OVX) group, except their ovaries were not removed. After 6 weeks, all animals were assessed for total body bone mineral density (BMD) using dual-energy X-ray absorptiometry (DXA; InAlyzer, Medikors, Seongnam, Korea) and $\mu$ CT (NFR Polaris-G90, NanoFocus Ray Co.) to confirm the usefulness of the OVX group as a model of osteoporosis. The animals were also weighed weekly.

Six weeks after the OVX or SHAM surgery, a femoral defect was created. The rats were divided into the following five groups: SHAM (sham-operation), OVX, OVX + Ti, OVX + Ti (1 mg) and OVX + Ti (2 mg). The OVX + Ti group received a Ti implant with only PLGA-coating, whereas the OVX + Ti (1 mg) and $\mathrm{OVX}+\mathrm{Ti}(2 \mathrm{mg})$ groups received a Ti implant with PLGA-coating containing $1 \mathrm{mg}$ and $2 \mathrm{mg}$ of E-2,5DHB, respectively.

The animals were anesthetized by an intramuscular injection of Zoletil and Rumpun. Both hind limbs of the rats were shaved. A longitudinal incision through the skin and the muscle was made on the medial surface of the femur. After exposure of the medial side of the distal femur, a bone defect $3 \mathrm{~mm}$ in diameter and $3 \mathrm{~mm}$ in depth was created perpendicular to the bone's surface. To create the defect, a dental drill 
(Marathon-N1, Saeyang Co., Daegu, Korea) was used with constant saline cooling. The defects were irrigated with normal saline to remove bone residue. Then, the drilled cavity was washed with saline and dried using a sterile cotton gauze. Defects were made bilaterally and transplants were randomized. The defect was either left empty (as the control) or the 3D printed porous Ti implants were positioned within the cavity. The subcutaneous tissue layer was closed with absorbable 4-0 sutures, after which the skin was closed. Finally, the defects were covered by returning the quadriceps to their original position and repositioning the patella.

\section{Dual-energy x-ray absorptiometry (DXA)}

Bone mineral content (BMC) and BMD of the femur were assessed by DXA. All femora were evaluated 6 weeks after the ovariectomy.

\section{$\mu \mathrm{CT}$ analysis}

At 4, 8 and 12 weeks following transplantation of $\mathrm{Ti}$ implants containing E-2,5-DHB into the OVX rats, the operated animals were scanned using a high resolution $\mu \mathrm{CT}$ imaging system. The animals were placed in a supine position onto the scan stage and sedated by inhalationof $2 \%$ isoflurane. The following $\mu \mathrm{CT}$ settings were used: resolution $39 \mu \mathrm{m}$, tube voltage $65 \mathrm{kV}$, tube current $115 \mu \mathrm{A}$ and exposure time $34 \mathrm{~ms}$ for each of the $360^{\circ}$ rotational steps. The reconstruction image size was $1024 \times 1024$ pixels and the number of slices was 512. 3D models of the trabecular bones of the femur were reconstructed using the Lucion software (Mevisys, Seoul, Korea) to evaluate bone alterations. The $\mu \mathrm{CT}$ sagittal section through the midline of the defect was selected to measure each defect.

\section{Histological analysis}

Histology was performed on the femora of each group of animals to study the bone-Ti interface and bone morphology. After harvesting the femora and removing the surrounding soft tissues, the bones were fixed in $10 \%$ neutral-buffered formalin solution, dehydrated in graded ethanol solutions ranging from $70 \%$ to $100 \%$ and then embedded in Technovit 7200 methacrylate resin (Heraeus Kulzer, Bad Sachsa, Germany). Sections of $40 \pm 5 \mu \mathrm{m}$ were obtained using an EXAKT diamond cutting system (EXAKT 300 CP, Bamberg, Germany) and stained with Goldner's trichrome to study mineralization. The stained sections were observed using an optical microscope (BX51, Olympus, Tokyo, Japan).

\section{Mechanism of osteoclast differentiation inhibition by E-2,5-DHB: western blot}

MC3T3-E1 mouse preosteoblast cells were maintained in a $\alpha$-MEM without ascorbic acid, supplemented with $10 \%$ FBS and $1 \%$ antibiotic and antimycotic solution. For all experiments, cells were used up to the 10 th passage. They were grown at $37^{\circ} \mathrm{C}$ in a $5 \%$ carbon dioxide atmosphere incubator. To evaluate osteoprotegerin (OPG) and receptor activator of nuclear factor-kappa B ligand (RANKL) expression, MC3T3-E1 cells were seeded onto 6-well plates at $2 \times 10^{5}$ cells/well density and cultured in the presence or absence of E-2,5-DHB at 5 and $10 \mu \mathrm{g} / \mathrm{mL}$. After being cultured for $7 \mathrm{~d}$, the cells were extracted by adding ice-cold radioimmunoprecipitation assay (RIPA) buffer. The cell lysates were centrifuged at $14,000 \times g$ for $15 \mathrm{~min}$ at $4{ }^{\circ} \mathrm{C}$. Protein determination was performed by Bradford assay according to the manufacturer's protocol. Protein samples were separated by electrophoresis using a $12.5 \%$ sodium dodecyl sulfate polyacrylamide (SDS-PAGE) gel and then transferred onto polyvinylidene difluoride membranes. The membranes were incubated overnight at $4{ }^{\circ} \mathrm{C}$ with a rabbit polyclonal antiOPG antibody (1 : 1000 dilution; ab183910, Abcam, Cambridge, UK), a mouse monoclonal anti-RANKL antibody (2 $\mu \mathrm{g} / \mathrm{mL}$; MAB6263, R\&D system, Minneapolis, MN, USA) or a rabbit polyclonal anti- $\beta$ actin antibody ( 1 : 1000 dilution; 4967S, Cell Signaling, Danvers, MA, USA). Finally, the membranes were incubated with a horseradish-peroxidase-conjugated secondary antibody [anti-mouse IgG (1 : 2000; 7076S, Cell Signaling) or anti-rabbit IgG (1 : 2000; 7074P2, Cell Signaling)]. Protein expression was detected by enhanced chemiluminescence (Cell Signaling). All bands were quantified using Image J software (National Institutes of Health, Bethesda, MD, USA).

\section{Statistical analysis}

All results are expressed as the mean \pm standard deviation (SD) of at least three determinations. Statistical analysis was performed by analysis of variance (ANOVA), using SPSS software (SPSS Inc. Chicago, IL, USA). Statistically significant values were defined as $p<0.05$.

\section{Results}

\section{Characterization of titanium implants containing E-2,5-DHB}

To determine the loading efficacy of E-2,5-DHB, the concentration of E-2,5-DHB coating the Ti implants was measured (Table 1). Ti implants containing E-2,5DHB were loaded with different amounts of E-2,5DHB. The loading concentrations of E-2,5-DHB for the $1 \mathrm{mg}$ and $2 \mathrm{mg}$ implants were $1.1 \mathrm{mg}$ and $1.9 \mathrm{mg}$, respectively.

The surface morphology of the Ti implants was observed after PLGA coating (Fig. 2a). The prepared Ti implants had porous structures with open micropores ranging from 200 to $250 \mu \mathrm{m}$. After being coated with PLGA, the Ti implants still maintained a large pore structure. Scanning electron microscopy (SEM) images of the Ti implants revealed that their pore walls exhibited a surface with regular micropores. After being coated by PLGA, 
a

Bare Ti

PLGA-coated Ti

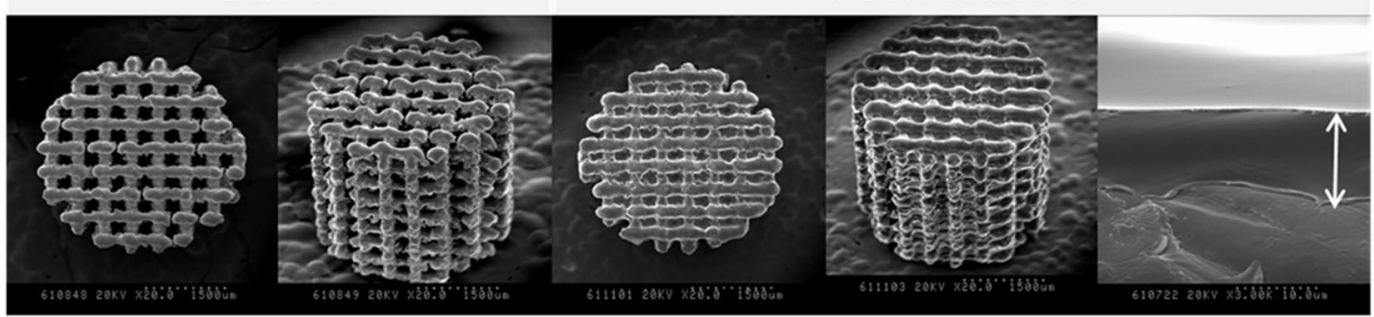

PLGA thickness

$(10 \pm 2 \mu \mathrm{m})$

b

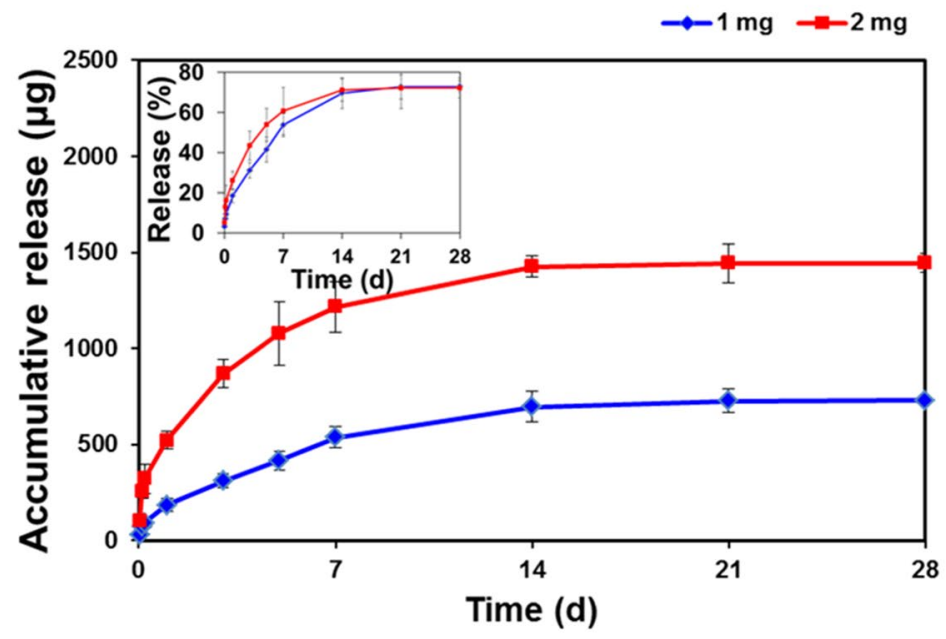

Fig. 2. Characterization of E-2,5-DHB-incorporating PLGA-coated Ti implants. (a) SEM images of Ti-only implants and PLGA-coated Ti implants. (b) E-2,5-DHB release kinetic from E-2,5-DHB-incorporating PLGA-coated Ti implants in PBS at $37^{\circ} \mathrm{C}$. The data are presented as mean $\pm \mathrm{SD}(n=4)$.

a

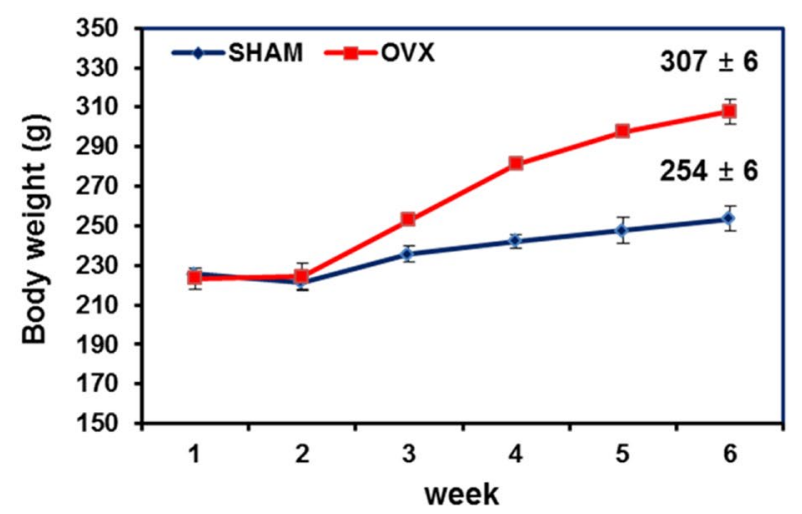

b

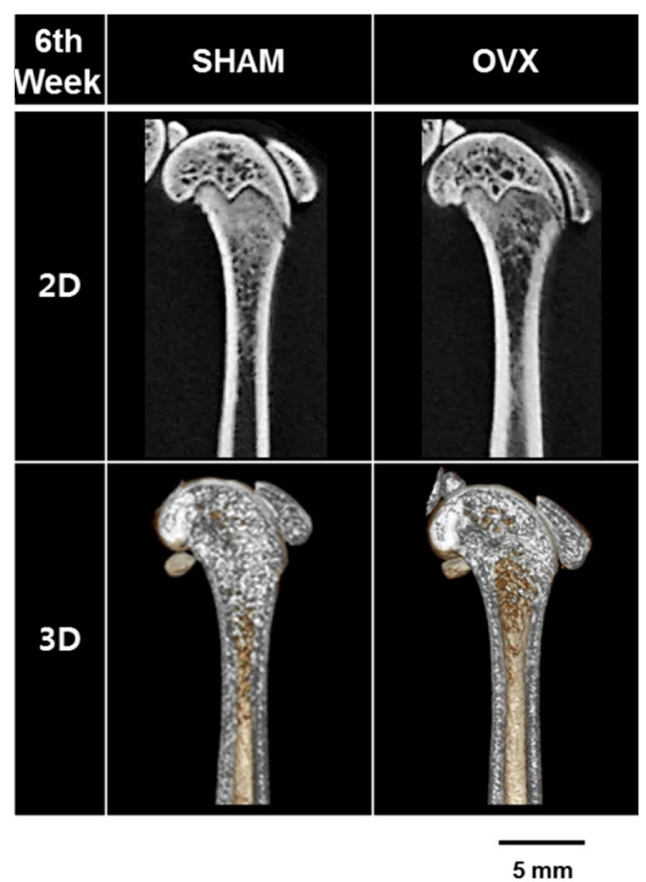

Fig. 3. Confirmation of the rat osteoporotic model. (a) The body weight of OVX rats was significantly larger than that of the SHAM control rats. (b) $\mu \mathrm{CT}$ : 2-dimensional (2D) and 3D images of distal femora from SHAM and OVX rats (scale bar $=500 \mu \mathrm{m}$ ) at 6 weeks. 
Table 2. Confirmation of the rat osteoporotic model. Femur BMC and BMD were significantly lower in the OVX group compared to the SHAM group. Data are shown as mean \pm SD.

\begin{tabular}{|c|c|c|}
\hline & SHAM & OVX \\
\hline BMC $(\mathbf{g})$ & $0.107 \pm 0.008$ & $0.101 \pm 0.017$ \\
\hline BMD $\left(\mathrm{g} / \mathrm{cm}^{2}\right)$ & $0.374 \pm 0.025$ & $0.347 \pm 0.015$ \\
\hline
\end{tabular}

the Ti implants still maintained this pore structure. The coated-PLGA thickness was approximately $10 \pm 2 \mu \mathrm{m}$. Release profiles obtained after the release of E-2,5-DHB from E-2,5-DHB-incorporating PLGAcoated Ti implants are shown in Fig. 2b. At day 7, the amount of E-2,5-DHB released from the implants containing 1 and $2 \mathrm{mg}$ of E-2,5-DHB was $54 \%$ and $61 \%$, respectively. E-2,5-DHB released from the E-2,5-DHB-incorporating PLGA-coated Ti implants exhibited a burst release, with most of the drug being released within the first $7 \mathrm{~d}$. Over the $28 \mathrm{~d}$ period, $70 \%$ of the E-2,5-DHB was released from both the 1 and $2 \mathrm{mg}$ E-2,5-DHB-incorporating PLGA-coated Ti implants, resulting in a sustained release profile.

\section{Characterization of the osteoporosis animal model}

Animals in the sham-operated group showed a steady increase in body weight during the 6 weeks observation period (Fig. 3a). Following ovariectomy, OVX rats weighed significantly more than SHAM rats at the evaluated time intervals. Initial body weights $(221 \pm 6 \mathrm{~g})$ were similar among all rats. At the end of the 6 weeks period of osteoporosis induction, the final body weight was significantly higher in OVX rats $(307 \pm 6 \mathrm{~g})$ than in SHAM rats $(254 \pm 6 \mathrm{~g})(p<0.01)$.

6 weeks after the initial surgery, the mean femoral BMC was significantly lower in the OVX group than in the SHAM group: $0.101 \pm 0.017 \mathrm{~g}$ and $0.107 \pm 0.008 \mathrm{~g}$, respectively $(p<0.05)$ (Table 2$)$. Likewise, the mean BMD of the femur was significantly lower in the OVX group $\left(0.347 \pm 0.015 \mathrm{~g} / \mathrm{cm}^{2}\right)$ than in the SHAM group $\left(0.374 \pm 0.025 \mathrm{~g} / \mathrm{cm}^{2}\right)(p<0.05)$.

$\mu \mathrm{CT}$ analysis revealed structural differences between the OVX and SHAM groups with respect to the trabecular architecture of the femur (Fig. 3b).

\section{Bone healing of femoral defects in ovariectomy rats}

$\mu \mathrm{CT}$ images were acquired to analyze the morphology and mineralization of new bone formation within the femoral defects. As shown in Fig. 4a (representative longitudinal $\mu \mathrm{CT}$ images), in the E-2,5-DHB group, newly formed bone was more than in the control group. This confirmed healing of the defects in the presence of E-2,5-DHB. Much less mineralized tissue was present in the untreated defects. A minimal amount of new bone formation was found in the only-PLGA-coated Ti group at all time intervals considered. The E-2,5-DHB-coated Ti groups exhibited the largest amounts of mineralized bone.

The bone newly formed in the Ti implant area after 3 months was assessed by PACS software, which measures bone density in Hounsfield units (HU) (Fig. 4b). Values obtained from $\mu \mathrm{CT}$ scans taken 4, 8 and 12 weeks postoperatively were compared and revealed the type of bone formed in the vicinity of the implant. Radiological evaluations using $\mu \mathrm{CT}$ scans showed a substantial increase in bone volume in the regions implanted with E-2,5-DHB after 12 weeks. The bone formed around the implant was more bone regeneration, with higher HU values. The enhancement of bone formation 8 and 12 weeks postoperatively in the E-2,5-DHB-coated Ti groups was superior (with higher HU values) compared to the only-PLGA-coated Ti group. HU values increased by $167 \%$ and $144 \%$ at 8 weeks and 12 weeks, respectively, in the E-2,5-DHB (1 mg) group and $154 \%$ and $144 \%$ in the E-2,5-DHB (2 mg) group. In the only-PLGA-coated group, the volume of bone formed was less compared to the successfully implanted E-2,5-DHB-coated group areas, with low $\mathrm{HU}$ values and signifying poor quality of formed bone. Moreover, as shown in Fig. 4c, no defect healing was observed in the defect area of osteoporotic rats during the transplantation period.

\section{Histology analysis}

Histologic findings (Goldner's trichrome staining) at the fracture sites supported the biomechanical evidence of accelerated defect healing by the released E-2,5-DHB (Fig. 5). Areas of newly formed bone, stained blue, differed considerably and only-PLGAcoated Ti implants showed less bone than E-2,5-DHBcoated Ti implants ( $1 \mathrm{mg}$ and $2 \mathrm{mg}$ ). Bone formation inside the porous space was mainly located at distal sites and resulted in direct bone-Ti contact. Direct bone-Ti contact was found throughout the entire bone-Ti interface. Trabecular bone areas around the E-2,5-DHB-coated Ti implants in animals were connected and more newly formed bone was seen in direct contact with the E-2,5-DHB coated surface compared with the only-PLGA-coated control implants.

Infiltration of newly formed bone was observed inside the pores filled with newly formed bone and within the Ti implants (Fig. 6a). Image J analysis showed that the result was not statistically significant, but the area of positive staining in animals with E-2,5DHB coated Ti implants was large. Quantification of the Goldner's trichrome staining images revealed differences between the only-PLGA-coated Ti group and the E-2,5-DHB-coated Ti groups, as the defects revealed less staining in the only-PLGAcoated control animals. The amount of bone around only-PLGA-coated Ti implants in osteoporotic rats 
a

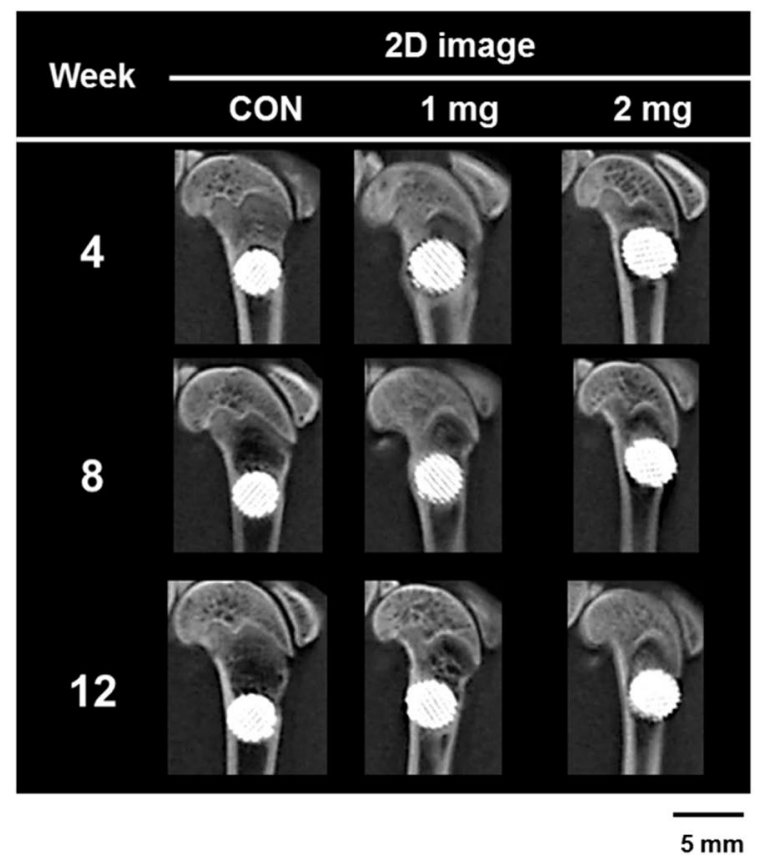

b

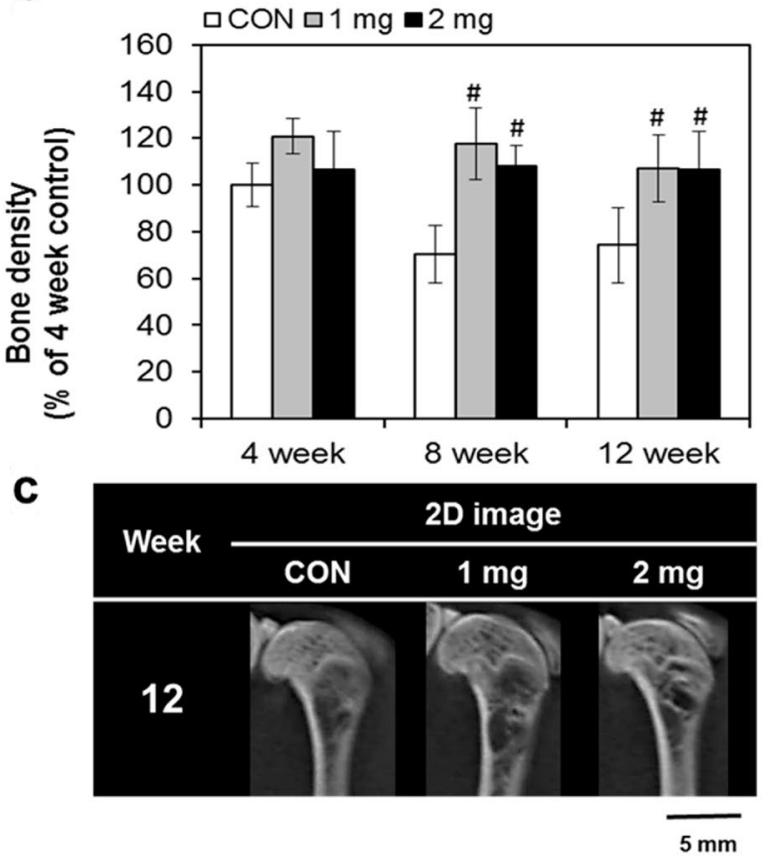

Fig. 4. Bone healing of femoral defects in OVX rats. (a) Representative $\mu \mathrm{CT}$ images of the distal femur showing mineralized bone formation within defects in the control (CON) group, titanium (Ti) + $1 \mathrm{mg}$ E-2,5-DHB group and $\mathrm{Ti}+2 \mathrm{mg}$ E-2,5-DHB group, 12 weeks after surgery. The CON group received a Ti implant coated with only PLGA. Scale bar $=5 \mathrm{~mm}$. (b) Quality of bone formed in the Ti implant area 3 months postoperatively was assessed by PACS software (Piviewer, INFINITT Healthcare, Seoul, Korea), which measures bone density in Hounsfield units. Data are shown as mean $\pm \mathrm{SD}\left({ }^{*} p<0.05\right.$ compared with the Ti group). (c) Representative $\mu \mathrm{CT}$ images of the distal femur showing mineralized bone formation within empty defect at 12 weeks. Scale bar $=5 \mathrm{~mm}$.

was smaller with respect to the E-2,5-DHB-coated groups (Fig. $6 \mathrm{~b}$ ). Statistically, the positive staining area percentage was significantly larger in the E-2,5DHB-coated Ti group than in the only-PLGA-coated Ti group.

\section{Inhibition of osteoclast differentiation by E-2,5- DHB}

E-2,5-DHB treatment inhibits osteoclast differentiation (Kwon et al., 2016). To investigate the mechanism of inhibition of osteoclast differentiation, OPG and RANKL expression were measured. The concerted actions of OPG, receptor activator of nuclear factorkappa B (RANK), and RANKL play a central role in maintaining the delicate balance between bone resorption and formation and in preserving bone mass (Boyle et al., 2013; Khosla et al., 2001; Hofbauer and Schoppet, 2004). To investigate the effect of E-2,5-DHB on OPG and RANKL expression, which are synthesized by osteoblasts, MC3T3-E1 cells were treated with E-2,5-DHB and OPG and RANKL protein levels were measured by western blot analysis (Fig. 7). The results indicated that expression of RANKL at the protein level was downregulated by E-2,5-DHB, whereas OPG levels were significantly upregulated. These results suggested that E-2,5-DHB ( 5 and $10 \mu \mathrm{g}$ / $\mathrm{mL}$ ) was more effective in stimulating the secretion of OPG as opposed to RANKL, thus exerting inhibitory effects on osteoclast differentiation.

\section{Discussion}

The aim of this study to investigate the effect of E-2,5-DHB on bone defects healing in osteoporotic conditions. The results confirmed the ability of E-2,5DHB-incorporating Ti implants using PLGA coating for local delivery of E-2,5-DHB to enhance fracture healing in osteoporotic bones.

Using conventional fabrication techniques, it is difficult to adjust the internal pore size, geometry and distribution (Pattanayak et al., 2009; Yang et al., 2000). In contrast, the SLM process is an effective technique for fabricating 3D porous structures directly from metal powders. Consequently, the process is suitable for designing artificial bone substitutes with complex inner and outer geometries that will fit a patient's defective bone. In this study, 3D printed Ti implants with pore sizes of 200 to $250 \mu \mathrm{m}$ were prepared. These implants were coated with a PLGA surface layer. PLGA coating did not decrease the pore size of the Ti implants. The small pore size benefits cell ingrowth and enhances diffusion of nutrients into the 3D scaffolds (Barou et al., 2002). Our results suggested that PLGA modification was a viable method to improve the physicochemical and drug-delivery properties of the Ti implants.

BMC and BMD at the femur site of OVX rats were shown to be decreased at week 6 post-surgery, when compared to the SHAM group. $\mu \mathrm{CT}$ images 
of the OVX rats confirmed a decrease in trabecular bone density, volume and thickness. OVX rats are commonly accepted as a suitable preclinical animal model for human postmenopausal osteoporosis (Jee and Yao, 2001; Lelovas et al., 2008; Turner, 2001). Similarly to previously published studies (Jee and Yao, 2001; Kalu, 1991; Lelovas et al., 2008; Turner, 2001), our results indicated that over the course of the experiment, OVX rats presented most of the characteristics observed in human postmenopausal osteoporosis. This included a significant decrease in trabecular bone thickness and mass and an increase in trabecular spacing, compared to healthy (SHAM group) animals.

Drugs can be administrated locally or systemically. Drugs delivered systemically are absorbed into the bloodstream and spread throughout the body by the circulatory system. This can lead to kidney and liver complications, lack of penetration into targeted tissues, systemic toxicity, as well as hospitalization of the patient. On the other hand, when the drug is delivered locally, the risk of side effects and overdosage, as for the systemic administration, may be limited and a high concentration of drug can effectively reach the target site (Price et al., 1996; Ruszczak and Friess, 2003; Somayaji et al., 1998). Local delivery is expected to increase the availability and efficacy of these bone anabolic or antiresorptive drugs and reduce the adverse effects associated with their tissue administration.

Reports of previous studies evaluate treatments to enhance new bone formation or suppress excessive bone resorption during bone regeneration, such as estrogen, parathyroid hormone (PTH), selective estrogen receptor modulators, strontium ranelate, calcitonin, bisphosphonates, sclerostin antibodies and Dickkopf-1 antibodies (Agholme et al., 2011; Cao et al., 2002; Li et al., 2007; Nozaka et al., 2008). However, antiosteoporotic drugs (i.e. bisphosphonates and RANKL inhibitors) often reduce new bone formation because they disrupt the linkage between osteoclast and osteoblast activity. PTH is an anabolic drug that can directly stimulate bone formation and, currently, it is the only anabolic agent approved for osteoporosis treatment by the Food and Drug Administration (Tashjian and Gagel, 2006). However, the anabolic effects of PTH are usually related to enhanced bone resorption and withdrawal of PTH associates with loss of its therapeutic effects (Tanizawa et al., 1998). Strontium ranelate is one of the agents reported to facilitate osteoporotic fracture healing, exhibiting the dual effects of promoting bone formation and inhibiting bone resorption (Bonnelye et al., 2008; Goldberg et al., 1985; Habermann et al., 2010). Results of the current study showed that E-2,5-DHB, similar to strontium ranelate, could also be used as an effective agent to promote bone fracture healing in osteoporotic conditions.

No signs of foreign body reactions or inflammatory responses were observed in our rat model. Porous titanium implants have recently received increased attention as a new biomaterial that can act as an osteoconductive scaffold, providing direct mechanical support in large bone defects at weight-bearing sites (Murr et al., 2010). However, they remain bioinert and, thereby, lack the ability to provide strong biological cues that enhance bone regeneration. This led to the hypothesis that E-2,5-DHB incorporated into porous

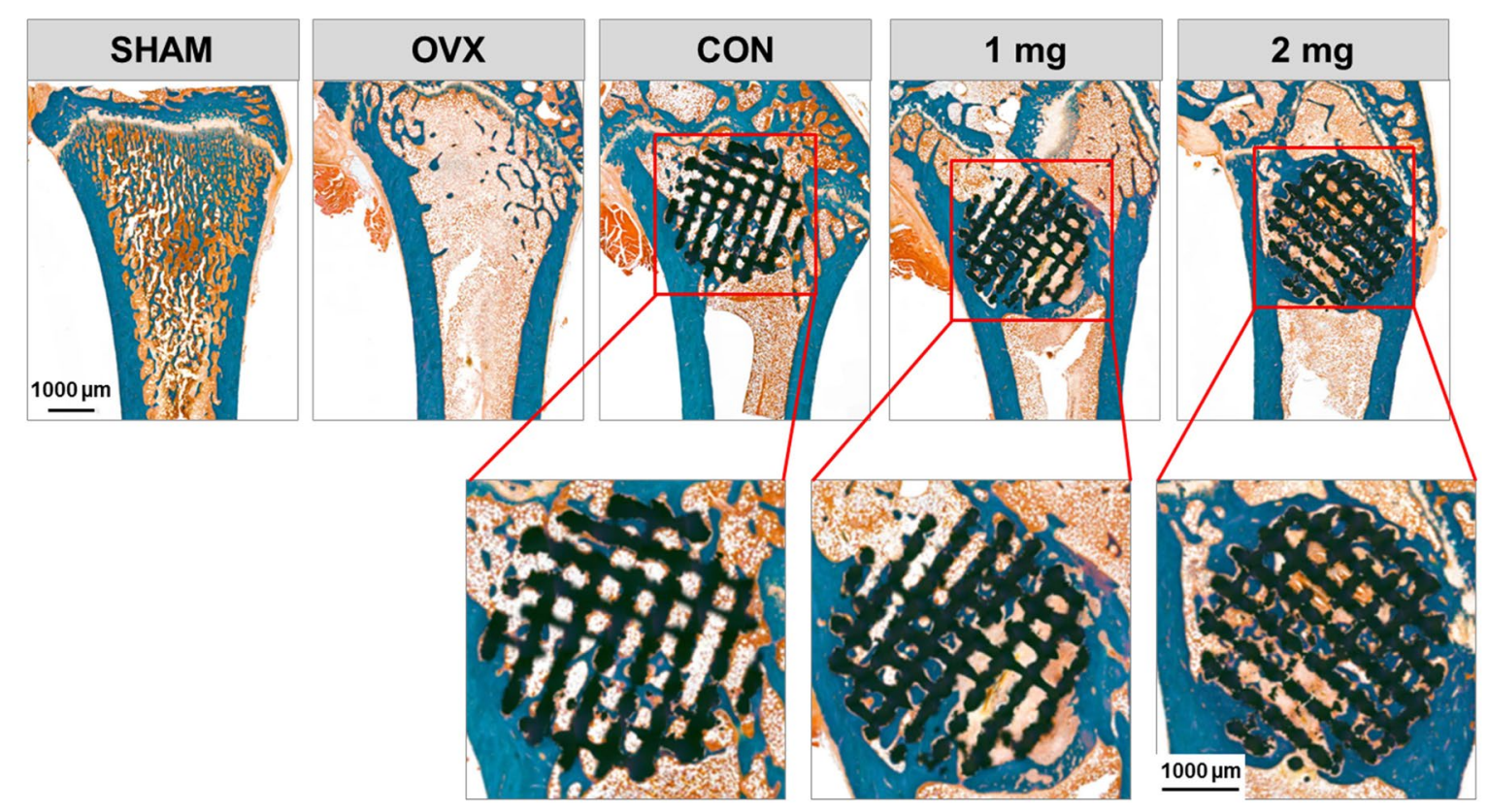

Fig. 5. Representative Goldner's trichrome staining of femur defects areas from SHAM, OVX and Ti implant groups at 12 weeks, respectively. The SHAM group underwent sham surgery; the OVX group underwent ovariectomy but received no implant; the Ti groups underwent ovariectomy and received implants containing PLGA coating only (CON group), $1 \mathrm{mg}$ of E-2,5-DHB in PLGA (1 mg group) or $2 \mathrm{mg}$ of E-2,5-DHB in PLGA (2 mg group). Scale bars $=1000 \mu \mathrm{m}$. 
Ti implants would result in a biologically active and mechanically strong composite biomaterial that would enhance bone healing of large bone defects.

Ideal orthopedic scaffolds should be biocompatible with surrounding cells to expedite satisfactory bone attachment between implants and surrounding bone structures (Borsari et al., 2009). It is well known that successful implant fixation is closely related to the parallelism of the bone structure near the implant and the thickness of the bone (Brånemark et al., 1997).

Additional bones formed around and near the implant, due to E-2,5-DHB elution, can be helpful in a wide range of orthopedic implant applications for increasing the reliability and accuracy of the device stability. Our results showed that local release of E-2,5-DHB directly from the implant had a positive effect on bone healing, while avoiding the disadvantages of systemic exposure.

Some herbal medicines are reported to be effective in treating various diseases. Local administration of 4-herb formula (CDNR) controls inflammation and promotes bone regeneration (Siu et al., 2015). Other studies report the prevention and treatment of osteoporosis using plant-derived natural products (Caichompoo et al., 2009; Jeong et al., 2005; Ha et al., 2003; Kim et al., 2008; Li et al., 2012; Suh et al., 2007). Lycii radicis cortex (LRC) extract may be a good natural herbal medicine candidate for the treatment of osteoporosis (Park et al., 2014) and for increasing spinal BMD (Wang et al., 2013). However, these studies evaluate the efficacy of herb extract as a drug to prevent osteoporosis and the effect of healing on osteoporotic defects is not evaluated. In the current study, unlike other herbal extras, E-2, 5-DHB showed a dual action on osteoblasts and osteoclasts, demonstrating bone defect healing effect in osteoporotic conditions.

Osteoclasts originate from the monocyte/ macrophage hematopoietic lineage and are responsible for bone resorption (Boyle et al., 2003; Zhao et al., 2007). RANKL, expressed by osteoblasts, plays a key role in the activation of osteoclasts by binding to the RANK receptor (Jimi et al., 1999). OPG, produced by osteoblasts, inhibits osteoclast differentiation by binding to RANKL (Hofbauer and Heufelder, 2000). The inhibitory effects of E-2,5-DHB on osteoclast differentiation are likely regulated by various paracrine factors, including OPG and RANKL, released from osteoblasts. In this study, it was demonstrated that E-2,5-DHB mediated an increase in the levels of secreted OPG, whereas it decreased RANKL levels. This suggested that E-2,5-DHB could affect the balance of OPG/RANKL pathway and promote bone healing in osteoporotic conditions. a
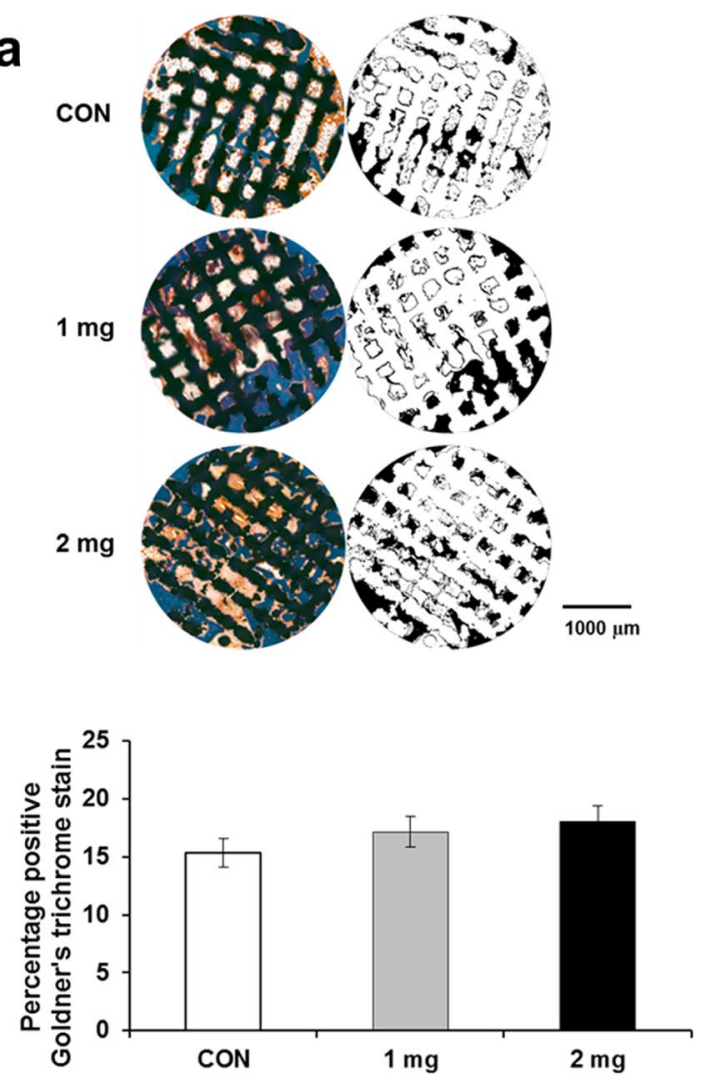

b
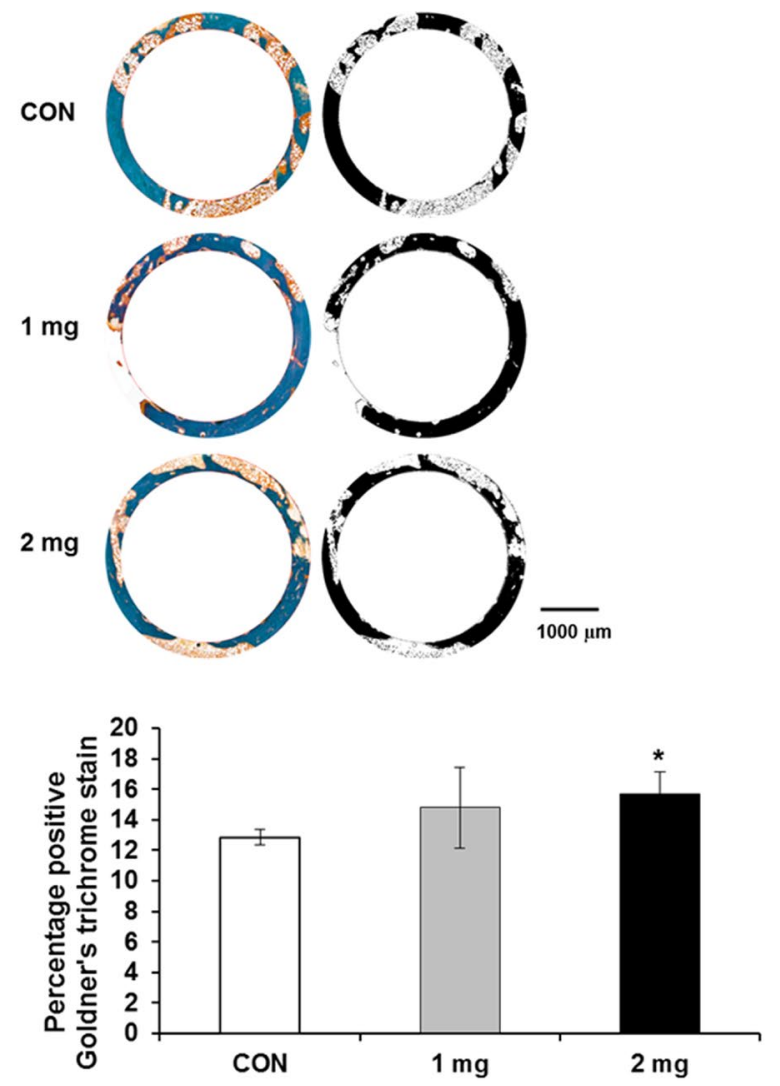

Fig. 6. Goldner's trichrome staining was analyzed by Image J software. For each sample, a circular region of the same size was chosen: (a) a circle with a diameter of $3 \mathrm{~mm}$ including the entire implant structure and (b) a 0.3 mm-thick ring of surrounding bone tissue. Data are shown as mean \pm SD. ${ }^{*} p<0.1$ compared to the Ti group in (b). 

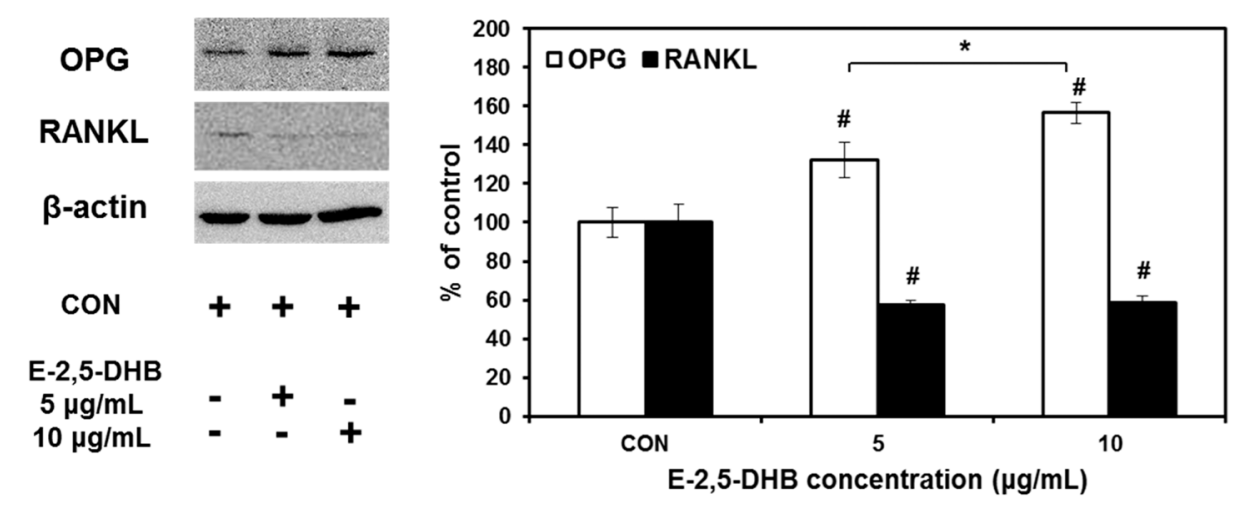

Fig. 7. Efficacy study of E-2,5-DHB evaluating osteoclast differentiation. Western blot of OPG and RANKL. Data are presented as mean \pm SD. *, \# statistically significant differences ${ }^{*} p<0.05$, $\# p<0.01)$. CON, control.

\section{Conclusion}

E-2,5-DHB-incorporating PLGA-coated Ti discs were prepared and whether this could influence osteoblast functions, as well as inhibit osteoclast differentiation, was evaluated. Subsequently, the local release of E-2,5-DHB from E-2,5-DHB-coated Ti implants was used to enhance bone formation around implants inserted into femoral defects. This study demonstrated that local treatment with E-2,5-DHB resulted in significantly more new bone formation in the rat femur deficient area compared to the control (PLGA-coated only Ti disc). Comparing the total new bone formation, it was revealed that both 1 and $2 \mathrm{mg}$ of E-2,5-DHB induced significantly more bone formation. Therefore, it appeared that E-2,5-DHBcoated Ti implants have great potential as an ideal local drug delivery system when using bone tissue scaffolds.

\section{Acknowledgements}

This study was supported by a faculty research grant of Yonsei University College of Medicine (6- 2016-0074) and the Bio \& Medical Technology Development Program of the NRF funded by the Korean government, MSIP (2017M3A9B3063638).

\section{References}

Agholme F, Isaksson H, Kuhstoss S, Aspenberg P (2011) The effects of dickkopf-1 antibody on metaphyseal bone and implant fixation under different loading conditions. Bone 48: 988-996.

Alho, A (1993) Mineral and mechanics of bone fragility fractures: a review of fixation methods. Acta Orthop Scand 64: 227-232.

Barou O, Mekraldi S, Vico L, Boivin G, Alexandre C, Lafage-Proust MH (2002) Relationships between trabecular bone remodeling and bone vascularization: a quantitative study. Bone 30: 604-612.

Barrios C, Brostrom LA, Stark A, Walheim G (1993) Healing complications after internal fixation of trochanteric hip fractures: the prognostic value of osteoporosis. J Orthop Trauma 7: 438-442.

Bonnelye E, Chabadel A, Saltel F, Jurdic P (2008) Dual effect of strontium ranelate: stimulation of osteoblast differentiation and inhibition of osteoclast formation and resorption in vitro. Bone 42: 129-138.

Borsari V, Fini M, Giavaresi G, Tschon M, Chiesa R, Chiusoli L, Salito A, Rimondini L, Giardino R (2009) Comparative in vivo evaluation of porous and dense duplex titanium and hydroxyapatite coating with high roughnesses in different implantation environments. J Biomed Mater Res Part A 89: 550-560.

Boyle WJ, Simonet WS, Lacey DL (2003) Osteoclast differentiation and activation. Nature 423: 337-342.

Brånemark R, Ohrnell LO, Nilsson P, Thomsen $P$ (1997) Biomechanical characterization of osseointegration during healing: an experimental in vivo study in the rat. Biomaterials 18: 969-978.

Caichompoo W, Zhang QY, Hou TT, Gao HJ, Qin LP, Zhou XJ (2009) Optimization of extraction and purification of active fractions from Schisandra chinensis (Turcz.) and its osteoblastic proliferation stimulating activity. Phytother Res 23: 289-292.

Cao Y, Mori S, Mashiba T, Westmore MS, Ma L, Sato M, Akiyama T, Shi L, Komatsubara S, Miyamoto K, Norimatsu H (2002) Raloxifene, estrogen, and alendronate affect the processes of fracture repair differently in ovariectomized rats. J Bone Miner Res 17: 2237-2246.

Cheng N, Dai J, Cheng X, Li S, Miron RJ, Wu T, Chen W, Zhang Y, Shi B (2013) Porous CaP/Silk composite scaffolds to repair femur defects in an osteoporotic model. J Mater Sci Mater Med 24: 19631975.

Do SH. Lee JW, Jeong WI, Chung JY, Park SJ, Hong IH, Jeon SK, Lee IS, Jeong KS (2008) Boneprotecting effect of Rubus coreanus by dual regulation of osteoblasts and osteoclasts. Menopause 15: 676-683.

Gardner MJ, Lorich DG, Lane JM (2004) Osteoporotic femoral neck fractures: management and current controversies. Instr Course Lect 53: 427439.

Goldberg VM, Powell A, Shaffer JW, Zika J, Bos GD, Heiple KG (1985) Bone grafting: role of histocompatibility in transplantation. J Orthop Res 3: 389-404. 
Ha H, Ho J, Shin S, Kim H, Koo S, Kim IH, Kim C (2003) Effects of Eucommiae cortex on osteoblastlike cell proliferation and osteoclast inhibition. Arch Pharm Res 26: 929-936.

Habermann B, Kafchitsas K, Olender G, Augat P, Kurth A (2010) Strontium ranelate enhances callus strength more than PTH 1-34 in an osteoporotic rat model of fracture healing. Calcif Tissue Int 86: 82-89.

Hofbauer LC, Heufelder AE (2000) The role of receptor activator of nuclear factor kappa-B ligand and osteoprotegerin in the pathogenesis and treatment of metabolic bone diseases. J Clin Endocrinol Metab 85: 2355-2363.

Hofbauer LC, Schoppet M (2004) Clinical implications of the osteoprotegerin/RANKL/RANK system for bone and vascular diseases. JAMA 292: 490-495.

Jee WS, Yao W (2001) Overview: animal models of osteopenia and osteoporosis. J Musculoskeletal Neuronal Interact 1: 193-207.

Jeong JC, Lee JW, Yoon CH, Lee YC, Chung $\mathrm{KH}, \mathrm{Kim}$ mg, Kim CH (2005) Stimulative effects of Drynariae rhizoma extracts on the proliferation and differentiation of osteoblastic MC3T3-E1 cells. J Ethnopharmacol 96: 489-495.

Jimi E, Akiyama S, Tsurukai T, Okahashi N, Kobayashi K, Udagawa N, Nishihara T, Takahashi N, Suda T (1999) Osteoclast differentiation factor acts as a multifunctional regulator in murine osteoclast differentiation and function. J Immunol 163: 434-442.

Kalu DN (1991) The ovariectomized rat model of postmenopausal bone loss. Bone Miner 15: 175-191.

Khosla S (2001) Minireview: the OPG/RANKL/ RANK system. Endocrinology 142: 5050-5055.

Kim KW, Suh SJ, Lee TK, Ha KT, Kim JK, Kim KH, Kim DI, Jeon JH, Moon TC, Kim CH (2008) Effect of safflower seeds supplementation on stimulation of the proliferation, differentiation and mineralization of osteoblastic MC3T3-E1 cells. J Ethnopharmacol 115: $42-49$.

Kwon BJ, Lee MH, Koo MA, Han JJ, Park JC (2014) Ethyl-3,4-dihydroxybenzoate with a dual function of induction of osteogenic differentiation and inhibition of osteoclast differentiation for bone tissue engineering. Tissue Eng Part A 20: 2975-2984.

Kwon BJ, Lee MH, Koo MA, Kim MS, Seon GM, Han JJ, Park JC (2016) Ethyl-2, 5-dihydroxybenzoate displays dual activity by promoting osteoblast differentiation and inhibiting osteoclast differentiation. Biochem Biophys Res Commun 471: 335-341.

Kyllönen L, D'Este M, Alini M, Eglin D (2015) Local drug delivery for enhancing fracture healing in osteoporotic bone. Acta Biomater 11: 412-434.

Lelovas PP, Xanthos TT, Thoma SE, Lyritis GP, Dontas IA (2008) The laboratory rat as an animal model for osteoporosis research. Comp Med 58: 424430.

Li TM, Huang HC, Su CM, Ho TY, Wu CM, Chen WC, Fong YC, Tang CH (2012) Cistanche deserticola extract increases bone formation in osteoblasts. J Pharm Pharmacol 64: 897-907.

Li X, Luo X, Yu N, Zeng B (2007) Effects of salmon calcitonin on fracture healing in ovariectomized rats. Saudi Med J 28: 60-64.

Long M, Rack HJ (1998) Titanium alloys in total joint replacement - a materials science perspective. Biomaterials 19: 1621-1639.

Mullen L, Stamp RC, Fox P, Jones E, Ngo C, Sutcliffe CJ (2009) Selective laser melting: a unit cell approach for the manufacture of porous, titanium, bone in-growth constructs, suitable for orthopedic applications. II. Randomized structures. J Biomed Mater Res Part B 92: 178-188.

Murr LE, Gaytan SM, Medina F, Lopez H, Martinez E, Machado BI, Hernandez DH, Martinez L, Lopez MI, Wicker RB, Bracke J (2010) Next-generation biomedical implants using additive manufacturing of complex, cellular and functional mesh arrays. Philos Trans R Soc A 368: 1999-2032.

Nozaka K, Miyakoshi N, Kasukawa Y, Maekawa S, Noguchi H, Shimada Y (2008) Intermittent administration of human parathyroid hormone enhances bone formation and union at the site of cancellous bone osteotomy in normal and ovariectomized rats. Bone 42: 90-97.

Park E, Jin HS, Cho DY, Kim J, Kim MC, Choi CW, Jin Y, Lee JW, Park JH, Chung YS, Huh D, Jeong SY (2014) The effect of Lycii radicis cortex extract on bone formation in vitro and in vivo. Molecules 19:1959419609.

Pattanayak DK, Fukuda A, Matsushita T, Takemoto M, Fujibayashi S, Sasaki K, Nishida N, Nakamura T, Kokubo T (2011) Bioactive Ti metal analogous to human cancellous bone: fabrication by selective laser melting and chemical treatments. Acta Biomater 7: 1398-1406.

Pattanayak DK, Matsushita T, Doi K, Takadama H, Nakamura T, Kokubo T (2009) Effects of oxygen content of porous titanium metal on its apatiteforming ability and compressive strength. Mater Sci Eng C 29: 1974-1978.

Pei L, Tontonoz P (2004) Fat's loss is bone's gain. J Clin Investig 113: 805-806.

Price JS, Tencer AF, Arm DM, Bohach GA (1996) Controlled release of antibiotics from coated orthopedic implants. J Biomed Mater Res 30: 281-286.

Ravichandran R, Ng CCh, Liao S, Pliszka D, Raghunath M, Ramakrishna S, Chan CK (2012) Biomimetic surface modification of titanium surfaces for early cell capture by advanced electrospinning. Biomed Mater 7: 015001.

Rodan GA, Martin TJ (2000) Therapeutic approaches to bone diseases. Science 289: 1508-1514.

Ruszczak Z, Friess W (2003) Collagen as a carrier for on-site delivery of antibacterial drugs. Adv Drug Delivery Rev 55: 1679-1698.

Sáenz de Viteri V, Fuentes E (2013) In titanium and titanium alloys as biomaterials. InTech, Rijeka, 5: 155-181. 
Siu WS, Ko CH, Lam KW, Wat E, Shum WT, Lau CB, Ko KM, Hung LK, Lau DT, Leung PC (2015) Evaluation of a topical herbal agent for the promotion of bone healing. Evid Based Complement Alternat Med 2015: 905270.

Sjostedt A, Zetterberg C, Hansson T, Hult E, Ekstrom L (1994) Bone mineral content and fixation strength of femoral neck fractures: a cadaver study. Acta Orthop Scand 65: 161-165.

Somayaji BV, Jariwala U, Jayachandran P, Vidyalakshmi K, Dudhani RV (1998) Evaluation of antimicrobial efficacy and release pattern of tetracycline and metronidazole using a local delivery system. J Periodontol 69: 409-413.

Stamp R, Fox P, Neill WO, Jones E, Sutcliffe C (2009) The development of a scanning strategy for the manufacture of porous biomaterials by selective laser melting. J Mater Sci Mater Med 20: 1839-1848.

Strømsøe K, Kok WL, Høiseth A, Alho A (1993) Holding power of the $4.5 \mathrm{~mm} \mathrm{AO/ASIF} \mathrm{cortex} \mathrm{screw}$ in cortical bone in relation to bone mineral. Injury 24: 656-669.

Suh SJ, Yun WS, Kim KS, Jin UH, Kim JK, Kim MS, Kwon DY, Kim CH (2007) Stimulative effects of Ulmus davidiana Planch (Ulmaceae) on osteoblastic MC3T3-E1 cells. J Ethnopharmacol 109: 480-485.

Tanizawa T, Yamamoto N, Takano Y, Mashiba T, Zhang L, Nishida S, Endo N, Takahashi HE, Fujimoto R, Hori M (1998) Effects of human PTH(1-34) and bisphosphonate on the osteopenic rat model. Toxicol Lett 102-103: 399-403.

Tashjian AH Jr, Gagel RF (2006) Teriparatide [human PTH(1-34)]: 2.5 years of experience on the use and safety of the drug for the treatment of osteoporosis. J Bone Miner Res 21: 354-365.

Thijs L, Verhaeghe F, Craeghs T, Humbeeck JV, Kruth JP (2010) A study of the microstructural evolution during selective laser melting of Ti6Al4V. Acta Mater 58: 3303-3312.

Turner AS (2001) Animal models of osteoporosisnecessity and limitations. Eur Cells Mater 1: 66-81.

Wang ZQ, Li JL, Sun YL, Yao M, Gao J, Yang Z, Shi Q, Cui XJ, Wang YJ (2013) Chinese herbal medicine for osteoporosis: a systematic review of randomized controlled trails. Evid Based Complement Alternat Med 2013: 356260.

Yang YZ, Tian JM, Tian JT, Chen ZQ, Deng XJ, Zhang DH (2000) Preparation of graded porous titanium coatings on titanium implant materials by plasma spraying. J Biomed Mater Res 52: 333-337.

Zhang Y, Miron RJ, Li S, Shi B, Sculean A, Cheng X (2012) Delivery of PDGF-B and BMP-7 by mesoporous bioglass/silk fibrin scaffolds for the repair of osteoporotic defects. Biomaterials 33: 66986708

Zhao Q, Shao J, Chen W, Li YP (2007) Osteoclast differentiation and gene regulation. Front Biosci 12: 2519-2529.

\section{Discussion with Reviewer}

Wing Hoi Cheung: What is the potential to translate E-2,5-DHB-incorporating titanium implant for the use in clinical application?

Authors: E-2,5-DHB-incorporating titanium implants showed promising clinical potential, enhancing implant performance in the osteoporotic bone condition. In recent year, there has been an increasing effort to restore bone defects in patients with osteoporosis. Also, E-2,5-DHB-incorporating titanium implants showed great potential for implant surface coating in several clinical applications, including dental, plastic and orthopedic surgery.

Wing Hoi Cheung: Is there any side effect of E-2,5DHB?

Authors: E-2, 5-DHB is a derivative of Rubus coreanus extract, which does not represent a serious concern for safety since it is used as health food supplements. No serious adverse events, such as foreign body reactions or inflammatory reactions, have been observed in our rat model. Nevertheless, any side effect during clinical applications need to be confirmed.

Editor's note: The Scientific Editor responsible for this paper was Chris Evans. 